\title{
Intraoperative Ear Injury, CTCAE
}

National Cancer Institute

\section{Source}

National Cancer Institute. Intraoperative Ear Injury, CT CAE. NCI Thesaurus. Code C143600.

A finding of damage to the ear during a surgical procedure. 